\title{
Uma Abordagem para Recomendação Automática e Dinâmica de Objetos de Aprendizagem Baseada em Estilos de Aprendizagem
}

\author{
Vitor C. de Carvalho ${ }^{1}$, Fabiano A. Dorça ${ }^{1}$, Renan G. Cattelan ${ }^{1}$, Rafael D. Araújo ${ }^{1}$ \\ ${ }^{1}$ Faculdade de Computação - Universidade Federal de Uberlândia (UFU) \\ Uberlândia - MG - Brasil \\ vitorcarvalho@comp.ufu.br, \{fabiano, renan\}@facom.ufu.br, rafael@doutorado.ufu.br
}

\begin{abstract}
The adaptation of content delivery in systems aimed at teaching is a research area in full expansion. This is explained by studies showing that students tend to have better performances when the content delivery is customized. In this context, students' learning styles should be observed, due to the importance of this feature to the adaptivity process in such systems. Thus, this paper presents an efficient approach for personalization of the teaching process. Our approach is based on the automatic mapping of students' learning styles characteristics to learning objects' metadata. Promising results, obtained through experiments, are presented and demonstrate the soundness of our proposal.
\end{abstract}

Resumo. A adaptação de fornecimento de conteúdo em sistemas voltados para o ensino é uma área de pesquisa em franca expansão. Isto é explicado por trabalhos que demonstram que estudantes tendem a ter um maior aproveitamento quando a apresentação do conteúdo é personalizada. Nesse contexto, os estilos de aprendizagem dos estudantes devem ser observados, sendo esta uma das mais importantes características a serem consideradas no processo de adaptatividade nesses sistemas. Dessa forma, este artigo apresenta uma abordagem eficiente para personalização do processo de ensino, que se baseia no mapeamento automático de características de estilos de aprendizagem de estudantes em metadados de objetos de aprendizagem. São apresentados resultados promissores, obtidos por meio de experimentos, que demonstram a validade da proposta.

\section{Introdução}

Um objeto de aprendizagem (OA) pode ser definido como qualquer entidade, digital ou não, que possa ser utilizado, reutilizado ou referenciado no ensino assistido por tecnologia [Wiley 2002]. Entende-se por material digital, diversos tipos de mídia - como textos, animações, vídeos, imagens, aplicações ou páginas Web - utilizados para fins educacionais, isoladamente ou em conjunto [Behar and Torrezzan 2009].

OAs precisam ser padronizados para garantir algumas propriedades como interoperabilidade e durabilidade. Surgiram, assim, algumas propostas de padronização, como é o caso do LTSC/IEEE Learning Object Metadata (LOM) [IEEE 2002]. A partir da definição dos metadados, consegue-se informações preciosas sobre como pode-se fazer uso dos OA e quais são as condições para que se possa usá-los. 
A adaptação de fornecimento de conteúdo em sistemas voltados para o ensino é uma área de pesquisa em franca expansão. Isto é explicado por trabalhos que demonstram que estudantes tendem a ter um maior aproveitamento quando a apresentação do conteúdo é personalizada. Estudos mostram que as estratégias pedagógicas relacionadas aos estilos de aprendizagem (EAs) dos estudantes contribuem para que a aprendizagem seja mais fácil. Além disso, o processo de aprendizagem é mais efetivo se o material educacional utilizado também estiver relacionado com os EAs [Haider et al. 2010]. Desta forma, os EAs dos estudantes devem ser observados, sendo esta uma das mais importantes características a serem consideradas no processo de adaptatividade nesses sistemas.

Neste cenário, este artigo apresenta uma abordagem eficiente para personalização do processo de ensino baseada no mapeamento automático de características de EAs em metadados de OAs. Para isto foi desenvolvido um modelo computacional que relaciona o padrão de metadados LOM e os EAs definidos por [Felder and Silverman 1988]. Um modelo probabilístico de EAs, previamente definido por [Dorça et al. 2012], é utilizado para que se possa levar em consideração as incertezas relacionadas às preferências do estudante no processo de recomendação de OAs. Resultados promissores, obtidos através de experimentos, demonstram a validade da proposta.

O restante do artigo encontra-se organizado da seguinte maneira: na Seção 2, são discutidos alguns trabalhos relacionados; na Seção 3, são apresentados os principais conceitos utilizados no trabalho; na Seção 4, é descrita a abordagem proposta; na Seção 5, são apresentados os resultados dos experimentos realizados; e, por fim, na Seção 6, são feitas as considerações finais e apresentados trabalhos futuros.

\section{Trabalhos Relacionados}

Diversos trabalhos tratam o problema de recomendação de conteúdo educacional. Em [Cazella et al. 2012], é apresentado um sistema que usa recomendação automática de OAs com base no conceito de competência. O sistema é capaz de filtrar e retornar os OAs relevantes para o aluno. No entanto, tal processo requer grande número de intervenções humanas, mesmo após passada a fase de escolha, e não é descrito como os OAs devem ser especificados em um repositório.

O trabalho de [Zaina et al. 2012] traz uma abordagem não-dinâmica que utiliza o modelo e-LORS de recomendação, em que, a partir de um repositório, é realizada a busca dos OAs de acordo com o perfil do estudante baseado em [Felder and Silverman 1988]. Em [Ferreira et al. 2012], é apresentado um sistema de recomendação que foi concebido da união de tecnologias de agentes de software conhecidos, fazendo-se também uma análise de desempenho.

O trabalho de [Júnior et al. 2012] apresenta uma abordagem baseada em um algoritmo genético que se adapta às necessidades dos estudantes e fornece os OAs adequados às características do contexto no qual eles se encontram. Em todo o trabalho, como é feita uma busca contextual dos OAs, uma nova análise é requerida a cada solicitação do estudante.

Em [Zaina et al. 2012], apesar de serem utilizados modelos bem definidos para representação dos perfis de estudante e metadados dos OAs, não considera as incertezas relacionadas à modelagem do perfil do estudante, conforme analisa-se em [Dorça et al. 2012]. 
Como resultado, tem-se, então, uma recomendação determinística de conteúdo, o que pode ter efeito bastante negativo à medida que o modelo do estudante possuir inconsistências.

Dos trabalhos apresentados, poucos consideram um padrão conhecido e certificado para representação de metadados de OAs. Isso dificulta sua recuperação e força sua readaptação, num trabalho muitas vezes manual. Desta forma, um diferencial da proposta ora apresentada é acrescentar aos modelos das pesquisas atuais uma relação entre o padrão de metadados IEEE LOM e as dimensões dos EAs [Felder and Silverman 1988], modelados de maneira probabilística. Assim, torna-se possível inferir probabilidades sobre os OAs e medir sua distância vetorial em relação ao perfil do estudante, o que resulta em uma recomendação de conteúdo educacional potencialmente mais eficaz, que leva em consideração padrões bem definidos, e as incertezas inerentes a este processo.

\section{Referencial Teórico}

A seguir, são feitas algumas considerações gerais sobre o modelo de Felder-Silverman e o padrão LOM, dado que ambos serviram de base para este trabalho.

\subsection{Modelo de Felder-Silverman}

Nos trabalhos de [Felder and Silverman 1988], percebeu-se que cada pessoa tem sua forma de receber e processar informações. A essa característica foi dado o nome de Estilos de Aprendizagem (EA). EAs não são mutuamente exclusivos, ou seja, uma pessoa pode ter afinidade com mais de um EA, embora apenas um deles geralmente predomine.

O modelo considera 4 dimensões, com 2 subdivisões cada, conforme Tabela 1. Uma subdivisão representa um EA dentro da dimensão e associa um significado de como é o estudante. É importante ressaltar que uma dimensão completa outra para conseguir representar melhor a forma com que cada indivíduo se comporta diante dos dados que lhe são expostos. Esse modelo é muito usado por prover um bom grau de adaptatividade aos perfis dos estudantes.

\subsection{Padrão LOM}

OAs têm como características principais: interoperabilidade, acessibilidade e durabilidade. Interoperabilidade é a capacidade de se compartilhar um mesmo OA entre sistemas com ferramentas distintas. Acessibilidade é a capacidade de utilizar os OA de forma remota. Durabilidade é a possibilidade de se utilizar um OA sem reprojeto ou recriação, mesmo quando houver mudança na base tecnológica. Existe ainda uma característica que afeta diretamente a criação, o uso e reuso de um OA: a chamada granularidade [Duval and Hodgins 2003]. Quanto menor for a granularidade de um OA, maior é seu potencial de reuso. O padrão LOM define 4 níveis de granularidade: nível 1 - menor agregação; nível 2 - uma coleção de objetos do nível 1; nível 3 - uma coleção de objetos do nível 2; e nível 4 - com maior granularidade.

Para que essas características se mantenham, é necessário que haja um padrão, como o LOM [IEEE 2002]. O LOM é composto por 68 elementos hierarquizados, divididos em 9 categorias, que descrevem a semântica dos metadados de um objeto de aprendizagem. A semântica de um elemento é determinada pelo seu contexto, ou seja, os elementos são afetados pelo seu pai, categoria em que está inserido ou, ainda, pelos elementos contidos na mesma categoria. 
Tabela 1. Dimensões e estilos de aprendizagem no modelo de Felder-Silverman.

\begin{tabular}{|c|c|c|}
\hline $\begin{array}{l}\text { Dimensao do Estilo de } \\
\text { Apredizagem }\end{array}$ & Tipo & Descrição \\
\hline \multirow{2}{*}{ Percepção } & Sensitivo (S) & $\begin{array}{l}\text { Sabe lidar com fatos, dados puros e experimentos, é paciente com } \\
\text { os detalhes, mas não gosta de complicações. }\end{array}$ \\
\hline & Intuitivo (I) & $\begin{array}{l}\text { Sabe lidar com principios e teorias, fica facilmente entediado } \\
\text { quando são apresentados muitos detalhes e tende a aceitar } \\
\text { complicaçốes. }\end{array}$ \\
\hline \multirow{2}{*}{ Canal de Entrada } & Visual (Vi) & $\begin{array}{l}\text { Se lembra bem do que viu: imagens, diagramas, tabelas temporais, } \\
\text { filmes, etc. }\end{array}$ \\
\hline & Verbal (Ve) & Se lembra do que escutou, leu ou disse. \\
\hline \multirow{2}{*}{ Processamento } & Ativo (A) & Aprende bem em grupo e com o material que tem em mãos. \\
\hline & Reflexivo (Re) & $\begin{array}{l}\text { Aprende melhor quando pensa e reflete sobre a informação dada a } \\
\text { ele. Trabalha melhor sozinho ou com no máximo mais uma pessoa. }\end{array}$ \\
\hline \multirow{2}{*}{ Entendimento } & Sequencial (Seq) & $\begin{array}{l}\text { Segue um raciocínio linear quando está resolvendo problemas e } \\
\text { pode trabalhar com material especifico uma vez que compreende } \\
\text { ele parcialmente ou superficialmente. }\end{array}$ \\
\hline & Global (G) & $\begin{array}{l}\text { Tem grandes intuições sobre a informação, pode ter dificuldade de } \\
\text { expor como chegou a certo resultado, precisa de uma visão geral. }\end{array}$ \\
\hline
\end{tabular}

\section{Abordagem Proposta}

Inicialmente, foi realizada a analise do padrão LOM em toda sua extensão. Identificouse, então, os campos mais relevantes do padrão LOM em relação às características do modelo de EAs definido por [Felder and Silverman 1988]: o campo Structure, em que verifica-se a existência de ligações do OA a outros OAs, bem como o mecanismo dessa ligação; Aggregation Level, que quantifica a granularidade atribuída ao OA; Format, no qual se informa quais formatos de mídia estão presentes no OA (vídeo, imagem, texto ou outro formato); Interactivity Type, que descreve como é a interação do estudante com o conteúdo (por exemplo, por meio de exercícios), se o OA é apenas expositivo (por exemplo, apenas com textos), ou uma mistura de ambos; Learning Resource Type, que serve como um complemento ao campo Format (por exemplo, sendo o OA descrito como formato de texto, esse campo especializa de qual tipo específico esse texto é, como uma narrativa, um questionário, entre outras opções dadas pelo padrão); Interactivity Level, que complementa Interactivity Type, mostrando uma graduação de quão interativo é o OA; Semantic Density, referindo-se à densidade semântica do OA, ou seja, qual volume de informação o OA contém e quão bem explicadas estão as mesmas (por exemplo, um texto pequeno pode trazer muitas informações de modo superficial, tendo assim uma alta densidade semântica, ou pode trazer poucas informações de forma detalhada, com baixo valor de densidade semântica); Difficulty, que tenta quantificar o nível de esforço requerido para se assimilar o conteúdo do OA (por exemplo, de simples ou fácil, à difícil ou complexo).

Esta analise é detalhada em [Resende 2013], onde também se apresenta o mapeamento entre características do LOM e dos EAs. Posteriormente, foram definidas regras no formato Prolog (Figura 1) para abstração do entendimento. A cada regra interpretada como verdadeira para determinado $\mathrm{OA} X$ em relação à um EA, é acrescentado um ponto à característica correspondente do $\mathrm{OA}$. $\mathrm{O}$ cabeçalho da regra especifica o EA a ser incrementado, enquanto o corpo da regra especifica os campos do LOM a serem analisados. 


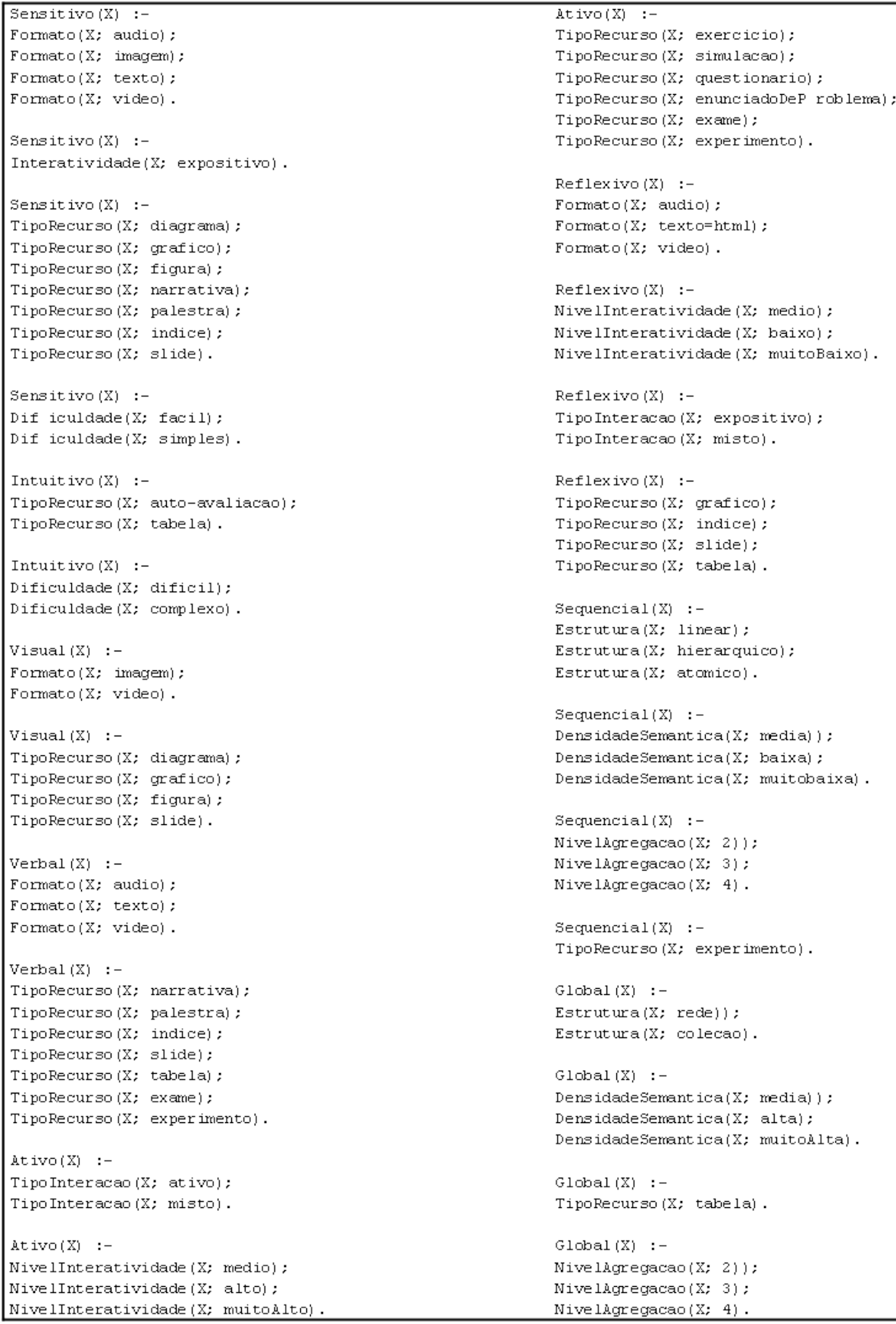

Figura 1. Listagem das regras para mapeamento dos campos do LOM em EAs. 
O campo "Formato" requer tratamento especial, pois pode assumir diversos valores. Como solução, foi escolhido um subconjunto do padrão [IANA 2014] para limitar as palavras-chave analisadas durante a recomendação de conteúdo. Com as pontuações adquiridas, são feitas combinações observando-se que, apesar de um OA ser predominantemente "Sensitivo" ou "Intuitivo", "Verbal" ou "Visual", "Ativo" ou "Reflexivo", e "Global" ou "Sequencial", tais EAs não são mutuamente exclusivos. Por exemplo, um OA como o apresentado na Figura 2 é descrito como ["Formato: áudio", "Interatividade: Expositivo", "Tipo de Recurso: Diagrama", "Dificuldade: complexo"\}, obtendo uma pontuação de 3 pontos no mapeamento para o EA "Sensitivo" e de 1 ponto no mapeamento para o EA "Intuitivo". Então, fazendo-se a conversão para proporcionalidades, pode-se dizer que esse OA é composto em $75 \%$ por características sensitivas, e em $25 \%$ por características intuitivas.

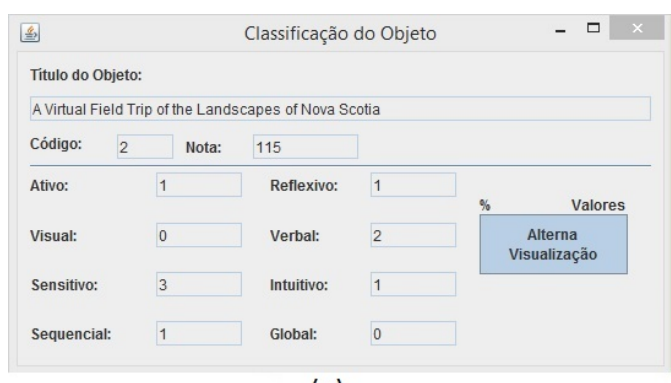

(a)

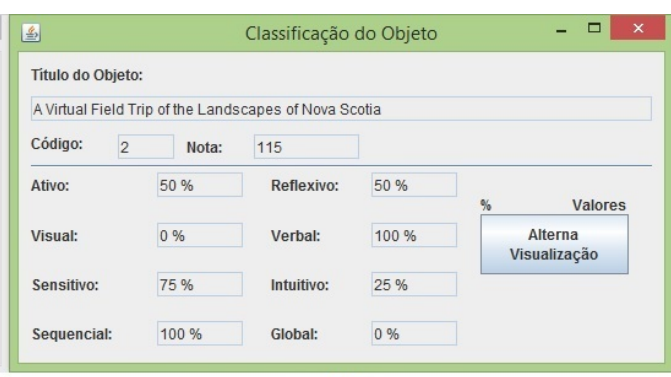

(b)

Figura 2. Exemplo de mapeamento das pontuações de um OA (a) para valores percentuais (b).

Em seguida, são obtidas as informações referentes ao perfil do estudante, modelado de maneira probabilística, conforme inicialmente proposto por [Dorça et al. 2013]. Então, dá-se início ao processo de recomendação automática dos OAs. A partir da soma dos valores absolutos da diferença entre as proporcionalidades das características do OA e do estudante, é calculada a distância entre os OAs e o modelo do estudante.

Tabela 2. Exemplo do cálculos da pontuação e da distância de um OA para determinado estudante.

\begin{tabular}{|c|c|c|c|c|c|c|c|c|}
\hline & \multicolumn{2}{|c|}{ Entrada } & \multicolumn{2}{c|}{ Processamento } & \multicolumn{2}{c|}{ Percepção } & \multicolumn{2}{c|}{ Entendimento } \\
\hline & Visual & Verbal & Ativo & Reflexivo & Sensitivo & Intuitivo & Sequencial & Global \\
\hline Estudante & 75 & 25 & 30 & 70 & 60 & 40 & 15 & 85 \\
\hline OA & 70 & 30 & 40 & 60 & 10 & 90 & 20 & 80 \\
\hline Diferença & \multicolumn{2}{|c|}{5} & \multicolumn{2}{|c|}{70} & \multicolumn{2}{c|}{50} & 5 \\
\hline Soma da diferença & \multicolumn{2}{|c|}{70} \\
\hline
\end{tabular}

$\mathrm{Na}$ Tabela 2, são exemplificadas a configuração de um perfil de estudante e a ordenação probabilística de um OA utilizando-se o método ora apresentado. Um OA com tal configuração teria uma distância de 70 em relação ao perfil do estudante, tendo maior probabilidade de melhor atender ao estudante do que um OA com distância superior a 70. Os OAs são, portanto, para efeito de recomendação, ordenados em ordem crescente de distância, ou seja, quanto menor a distância, melhor, resultando em um posicionamento mais acima na ordenação. 


\section{Resultados de Experimentos}

Para realização dos experimentos foi criado um protótipo que, dado um perfil de estudante, gera uma ordem de recomendações dos OAs aderentes. A Figura 3 apresenta a interface do protótipo.

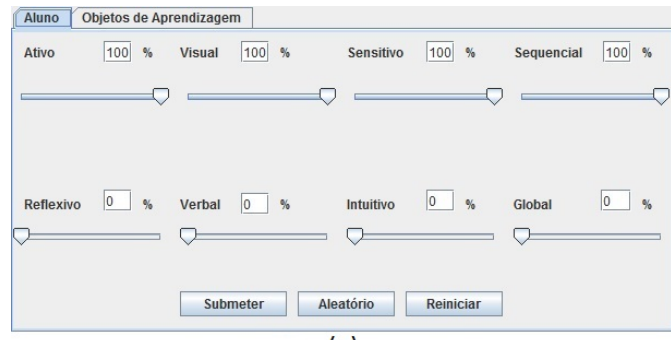

(a)

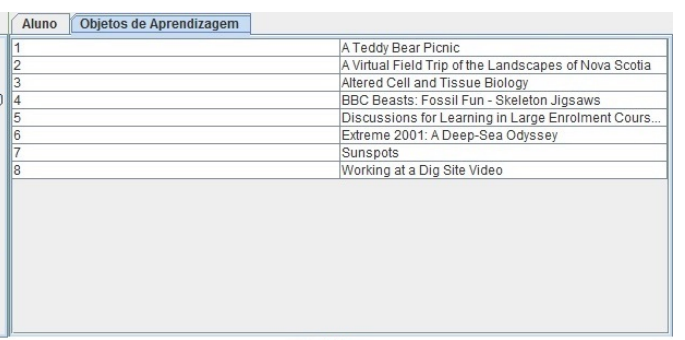

(b)

Figura 3. Representação de um perfil de estudante no protótipo criado.

Na Figura 3(a), é ilustrada a representação dos EAs do estudante. Na Figura 3(b) é apresentada a aba do protótipo com a listagem dos códigos e nomes dos OAs cadastrados. Essa informação é representada no sistema por meio de documentos XML, promovendo portabilidade e extensibilidade. Os experimentos foram realizados da seguinte maneira: primeiramente, configurava-se um perfil de estudante com as probabilidades de preferências por EAs, conforme descrito por [Dorça et al. 2013]. Em seguida, os resultados obtidos eram analisados considerando-se (1) se a similaridade entre a classificação de um OA e o perfil do estudante era mais próxima que os OAs em posições inferiores da lista ordenada; (2) se havia alguma mudança ao se alterar a configuração de EA do estudante, mantendo-se a mesma predominância de preferências.
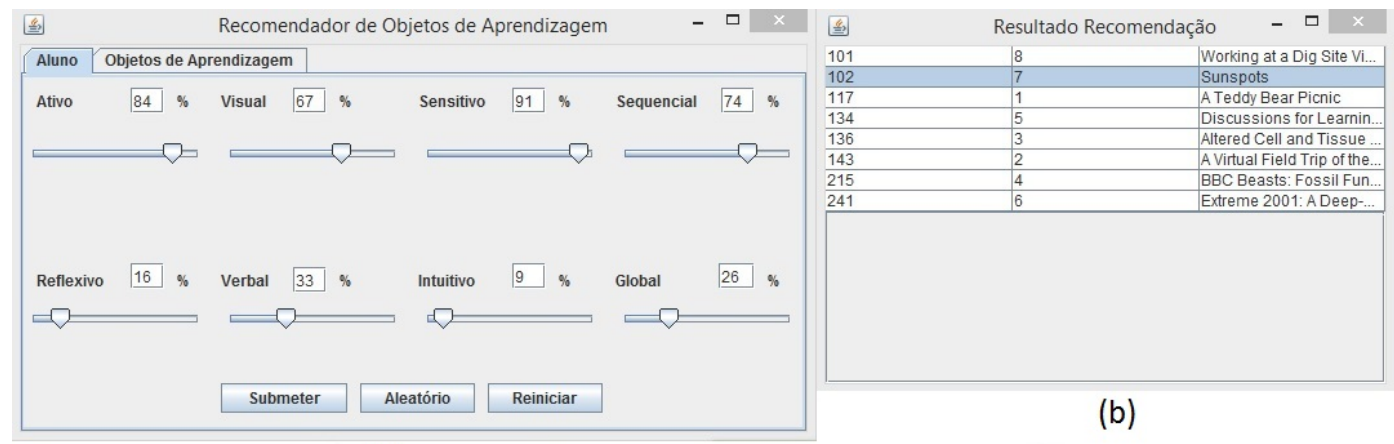

(a)

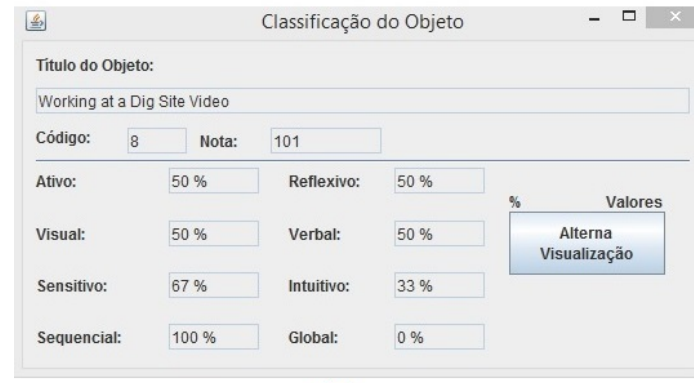

(c)

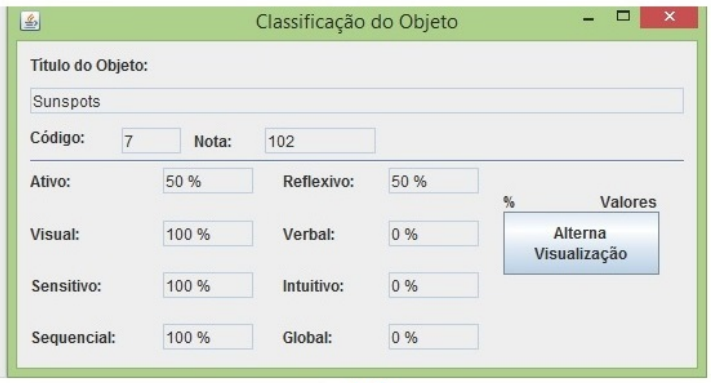

(d)

Figura 4. Exemplo de resultado obtido a partir da execução do protótipo. 
Na Figura 4(a), está apresentado um exemplo de perfil de estudante, e em 4(b), a lista de OAs ordenada (a primeira coluna da tabela mostra a pontuação que cada OA recebeu). $\mathrm{Na}$ 4(c) são visualizadas as proporcionalidades de composição do OA da primeira posição da lista. $\mathrm{Na}$ 4(d) o mesmo ocorre para o OA da segunda posição. Para realização dos testes, foram empregados OAs fictícios. Todas as 16 combinações possíveis de perfis de EAs foram testadas [Dorça et al. 2013, Dorça et al. 2012]. Cada qual com diferentes variações em seus percentuais para o mesmo conjunto de OAs, de modo a se analisar melhor os resultados obtidos. Os resultados mostraram-se promissores.

Notou-se que ao se manter a preferência do estudante por determinados EAs, variações nos valores das probabilidades destas preferências fazem com que alguns OAs variem consideravelmente em sua ordem. Como o modelo não leva em consideração apenas as proporcionalidades de composição de EAs nos OAs, mas sim a distância existente entre estas proporcionalidades e os valores das probabilidades de preferências do estudante, uma nova ordenação pode ser obtida sempre que o valor destas probabilidades forem recalculados (conforme descrito em [Dorça et al. 2013]). Esta atualização ocorreria constantemente em um curso, possibilitando uma recomendação automática e dinâmica conforme alterações minímas no perfil do estudante são realizadas. Esse tipo de comportamento pode ser observado na Figura 5, em que variações no perfil do estudante - (a), (b) ou (c) - alteram a classificação dos OAs e, consequentemente, a recomendação.

\section{Conclusão}

Neste artigo, foi apresentada uma abordagem para personalização do processo de ensino de acordo com os EAs dos estudantes. A abordagem proposta leva em consideração as preferências de EAs dos estudantes, e para isto faz a relação entre o padrão de metadados LOM e os EAs definidos por [Felder and Silverman 1988]. Como consequência, tem-se como contribuição principal deste trabalho um modelo computacional que relaciona EAs a OAs, e que através de um modelo probabilístico de modelagem de EAs (previamente definido em [Dorça et al. 2013]) consegue recomendar automática e dinamicamente OAs durante o processo de ensino, promovendo sua recuperação e ordenação de acordo com os EAs do estudante.

Com base na realização de experimentos, a proposta foi validada e mostrou-se potencialmente eficaz, podendo ser utilizada em ambientes virtuais de aprendizagem que forneçam um modelo do estudante baseado em EAs [Felder and Silverman 1988]. Para isso, porém, depende de certo compromisso por parte dos autores de conteúdo no preenchimento dos metadados que descrevem os OAs, dado que alguns campos são obrigatórios para se obter precisão na ordenação. Como trabalho futuro, pretende-se integrar o modelo a um ambiente virtual de aprendizagem para testes com OAs e estudantes reais. Assim, o modelo poderá ser usado para recuperação de OAs em repositórios existentes.

\section{Agradecimentos}

Os autores agradecem às agências brasileiras de pesquisa CNPq, CAPES e FAPEMIG, assim como à FACOM/UFU e PROPP/UFU, pelo apoio concedido a este trabalho. 


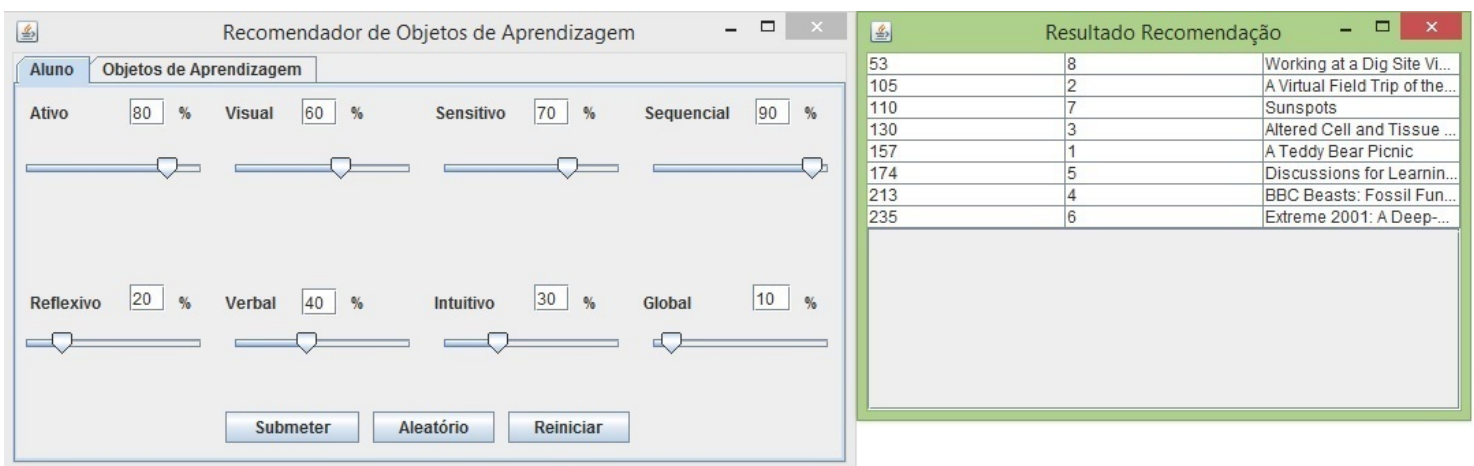

(a)

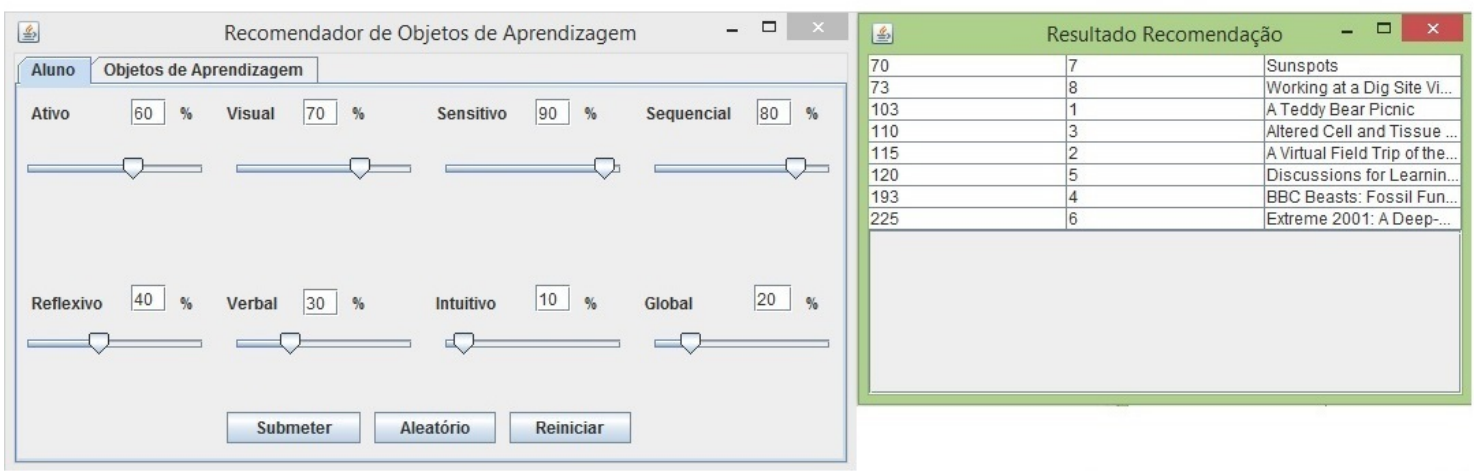

(b)

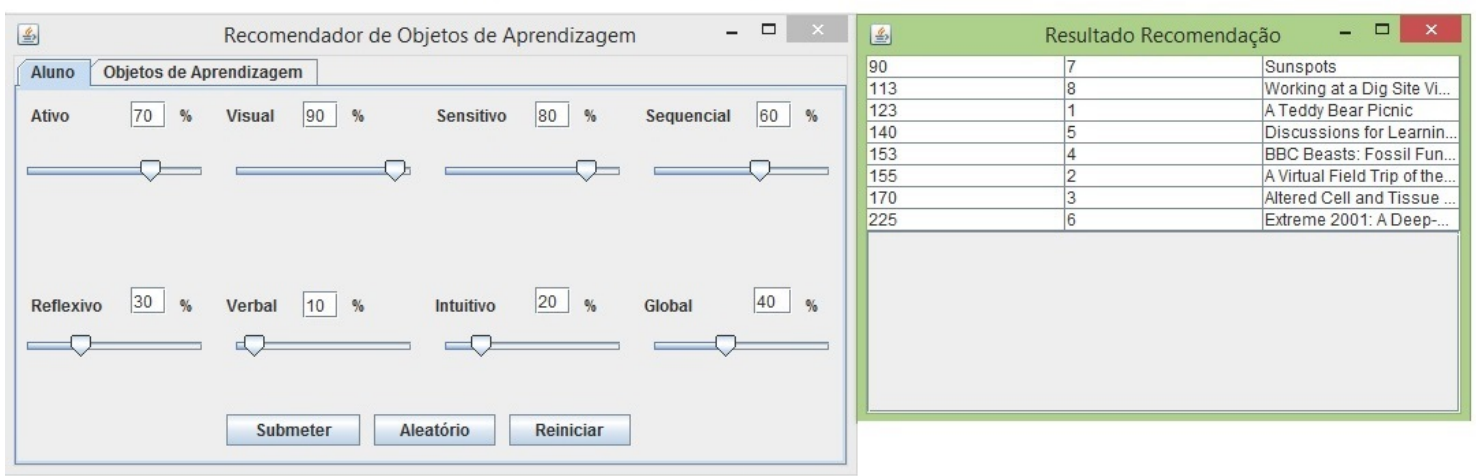

(c)

Figura 5. Exemplo de variação na ordem dos OAs conforme valores configurados para o perfil do estudante. 


\section{Referências}

Behar, P. A. and Torrezzan, C. A. W. (2009). Parâmetros para a construção de materiais educacionais digitais do ponto de vista do design pedagógico. In Modelos Pedagógicos em Educação a Distância. Artmed, Porto Alegre.

Cazella, S. C., Behar, P., Schneider, D., da Silva, K. K., and Freitas, R. (2012). Desenvolvendo um sistema de recomendação de objetos de aprendizagem baseado em competências para a educação: relato de experiências. In Anais do Simpósio Brasileiro de Informática na Educação, volume 23.

Dorça, F. A., Lima, L. V., Fernandes, M. A., and Lopes, C. R. (2013). A new approach to discover students learning styles in adaptive educational systems. Revista Brasileira de Informática na Educação, 21(01):76.

Duval, E. and Hodgins, W. (2003). A lom research agenda. In WWW2003, May 20?24, 2003, Budapest, Hungary.

Felder, R. M. and Silverman, L. K. (1988). Learning and teaching styles in engineering education. Engineering education, 78(7):674-681.

Dorca, F. A., Lima, L. V., Fernandes, M. A. and Lopes, C. R. (2012). A stochastic approach for automatic and dynamic modeling of studentś learning styles in adaptive educational systems. Informatics in Education-An International Journal, 11(2):191212.

Ferreira, L. G. A., Gluz, J. C., and Barbosa, J. L. V. (2012). Um modelo multiagente para recomendação de conteúdo educacional em um ambiente ubíquo. In Anais do Simpósio Brasileiro de Informática na Educação, volume 23.

Haider, M. T. U., Sinha, A. K., and Chaudhary, B. D. (2010). An investigation of relationship between learning styles and performance of learners. Intern. Journal of Engineering Science and Technology, 2(7):2813-2819.

IANA (2014). IANA Media Types. http://www.iana.org/assignments/media-types/mediatypes.xhtml.

IEEE (2002). 1484.12.1-2002 IEEE Standard for Learning Object Metadata.

Júnior, L. J., Mendes Neto, F. M., and da Silva, L. C. N. (2012). Uma abordagem baseada em algoritmo genético para recomendação de objetos de aprendizagem sensível ao contexto do estudante. In Anais do Simpósio Brasileiro de Informática na Educação, volume 23 .

Resende, D. (2013). Personalização em sistemas de ensino à distância com base no mapeamento de estilos de aprendizagem em objetos de aprendizagem. Trabalho de Conclusão de Curso. Bach. em Sistemas de Informação. Faculdade de Computação, Universidade Federal de Uberlândia - Minas Gerais.

Wiley, D. (2002). Connecting learning objects to instructional design theory: A definition, a metaphor, and a taxonomy, pages 3-23. The Agency for Instructional Technology, Bloomington, Indiana, USA.

Zaina, L. A., Bressan, G., Cardieri, M. A. A., and Rodrigues Júnior, J. F. (2012). e-lors: Uma abordagem para recomendacao de objetos de aprendizagem. Revista Brasileira de Informática na Educação, 20(1):04. 